\title{
Hydration dynamics gives the distinctive brown color in the "brown ring" nitrate test
}

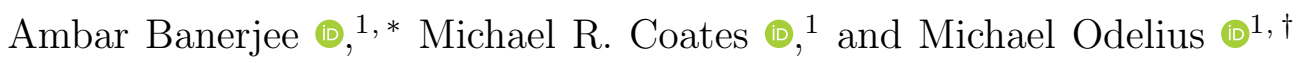 \\ ${ }^{1}$ Department of Physics, Stockholm University, \\ AlbaNova University Center, SE-106 91 Stockholm Sweden
}

\begin{abstract}
The brown ring test is one of the most popular and visually appealing reagent tests, commonly known to chemistry undergrads and familiar even to school students. The exact composition, mechanism and structure of the complex has been investigated for nearly a century. Recent studies have elucidated its UV-vis, EPR and Mössbauer spectra, mechanistic details and kinetics, followed by crystallization and structure determination in solid state. Nonetheless these studies were unable to address the aspects of solution structure and dynamics of the brown ring complex. We have conducted ab initio molecular dynamics simulations of the classic brown ring complex in aqueous solution. In the process from the simulation trajectory, we have identified that the classically established pseudo-octahedral $\left[\mathrm{Fe}\left(\mathrm{H}_{2} \mathrm{O}\right)_{5}(\mathrm{NO})\right]^{2+}$ complex is in chemical equilibrium with the square-pyramidal $\left[\mathrm{Fe}\left(\mathrm{H}_{2} \mathrm{O}\right)_{4}(\mathrm{NO})\right]^{2+}$ complex through the exchange of one of the coordinated $\mathrm{H}_{2} \mathrm{O}$ molecules. The dynamics in aqueous solution between the penta-aqua and tetra-aqua complexes in the brown ring system has to our knowledge never been suggested earlier. Interestingly we find, using ab initio multi-reference quantum chemical methods i.e. CASSCF/NEVPT2 and CASPT2 calculations, that the mixture of these two complexes is what gives the distinctive brown coloration to the brown ring test. We show that its UV-vis spectrum can be theoretically reproduced only by accounting these two species, and not solely the classically established $\left[\mathrm{Fe}\left(\mathrm{H}_{2} \mathrm{O}\right)_{5}(\mathrm{NO})\right]^{2+}$ complex. The energetics of the penta-aqua and tetra-aqua complexes is also investigated at the level of multi-reference quantum chemical methods.
\end{abstract}

* ambarpchem@gmail.com

$\dagger$ odelius@fysik.su.se 


\section{INTRODUCTION}

The brown ring test is a standard procedure in undergraduate and high school chemistry laboratory to detect the presence of nitrates in aqueous solution. The addition of $\mathrm{H}_{2} \mathrm{SO}_{4}$ to a solution containing nitrate ions, in presence of $\mathrm{Fe}^{2+}$ ions, produces a distinct brown ring at the interface of the two aqueous solutions. The green-brown color of the brown ring is traditionally attributed to the formation of the $\left[\mathrm{Fe}\left(\mathrm{H}_{2} \mathrm{O}\right)_{5}(\mathrm{NO})\right]^{2+}$ complex $[1]$, which has a quartet $(\mathrm{S}=3 / 2)$ ground state. This is considered to be a very unstable complex, degraded by light and heat, and also the presence of sulfate ions. This particular reaction and the chemistry behind it has been studied for more than a century [1-3]. This inherent instability has hindered a detailed study of the brown ring test, though recent attempts have given invaluable insights into the electronic structure and instability of the complex [4]. Wanat and co-workers have studied the kinetic and spectroscopic aspects of the reversible reaction of $\mathrm{NO}$ with $\left[\mathrm{Fe}\left(\mathrm{H}_{2} \mathrm{O}\right)_{6}\right]^{2+}$ to give the brown ring complex [4. Their study also indicated the presence of a $\mathrm{S}=3 / 2$ (quartet) species using EPR, which also also the presence of a complex with an electronic structure resembling that of a $\{\mathrm{FeNO}\}^{7}$ species, following the Enemark-Feltham notation [5]. The superscript in $\{\mathrm{FeNO}\}^{7}$ denotes the combined number of valence electrons in the $\mathrm{Fe} 3 d$ and $\mathrm{NO}\left(\pi^{*}\right)$ orbitals. Mössbauer spectroscopy in agreement with EPR study also indicated the presence of a $\{\mathrm{FeNO}\}^{7}$ species with a high spin $\mathrm{Fe}^{\mathrm{III}}$ center anti-ferromagnetically coupled with a $\mathrm{NO}^{-}(\mathrm{S}=1)$ moiety. Eventually Monsch and co-workers reported the crystal data for the $\left[\mathrm{Fe}\left(\mathrm{H}_{2} \mathrm{O}\right)_{5}(\mathrm{NO})\right]^{2+}$ complex in their recent study [6]. The crystal could be isolated due the use of bulky gallate counter ion having perfluoropinacolato ligands. The XRD established the theoretically predicted octahedral structure of the complex with an axial NO ligand. However, the crystal structure predicted by Monsch and co-workers gives information of the complex in the solid phase and is unable to shed light in the solution phase chemistry, which was also addressed in a very limited scope by earlier works like that of Wanat and co-workers [4].

The interest in the brown ring complex is not limited to only undergraduate text books and as a test for nitrate ions. The Fe-NO bond is of prime interest to the bio-inorganic chemists [7, 8]. NO binding to relevant Fe centers in metallo-enzymes has been the topic of research on both experimental and theoretical fronts [9-12. Traditional inorganic chemists have also found the Fe-NO bond very interesting, as reflected by the detailed study on 
Hieber's anion [13, 14]. Theoretical attempts have uni-vocally established the multiconfigurational character of the Fe-NO bond [15]17]. Pierloot and co-workers have also stressed upon the importance of dynamical correlation in description of the Fe-NO bond [15]. Multi-reference quantum chemical computations have also been used to show the $\mathrm{O}_{2}$ activation by Dinitrosyl Iron Complexes (DNICs) which contains two Fe-NO bonds [17]. The first attempt in using multireference theories to describe the classic brown ring complex was only done by Monsch and co-workers in 2019 [6]. Monsch and co-workers also performed various $a b$ initio and density function theory (DFT) computations highlighting the oxidation state and spin densities of the complex.

Here in this study we perform ab initio molecular dynamics (AIMD) simulations of the $\left[\mathrm{Fe}\left(\mathrm{H}_{2} \mathrm{O}\right)_{5}(\mathrm{NO})\right]^{2+}$ complex in aqueous environment combined with computations on cluster models for the UV-vis spectrum with multi-reference quantum chemical methods. The AIMD simulation suggests the formation of a $\left[\mathrm{Fe}\left(\mathrm{H}_{2} \mathrm{O}\right)_{4}(\mathrm{NO})\right]^{2+}$ species which remains in equilibrium with $\left[\mathrm{Fe}\left(\mathrm{H}_{2} \mathrm{O}\right)_{5}(\mathrm{NO})\right]^{2+}$. Based on this, high level multi-reference computations were performed to validate the observation from the dynamics. We conclude that energetically both theses species are accessible and that hydrogen $(\mathrm{H}-)$ bonding with surrounding water molecules dictates the stabilization of one species over the other. Serendipitously while investigating their electronic properties, we find that $\left[\mathrm{Fe}\left(\mathrm{H}_{2} \mathrm{O}\right)_{5}(\mathrm{NO})\right]^{2+}$ alone cannot reproduce the UV-vis spectra of the brown ring complex as published by Wanat and co-workers 44. We find that only the presence of $\left[\mathrm{Fe}\left(\mathrm{H}_{2} \mathrm{O}\right)_{4}(\mathrm{NO})\right]^{2+}$ along with $\left[\mathrm{Fe}\left(\mathrm{H}_{2} \mathrm{O}\right)_{5}(\mathrm{NO})\right]^{2+}$, together can account for all the peaks in the VIS region of the spectra. Hence the brown color of the brown complex is, according to our computations, the result of having a chemical equilibrium between $\left[\mathrm{Fe}\left(\mathrm{H}_{2} \mathrm{O}\right)_{5}(\mathrm{NO})\right]^{2+}$ and $\left[\mathrm{Fe}\left(\mathrm{H}_{2} \mathrm{O}\right)_{4}(\mathrm{NO})\right]^{2+}$ in solution. Different means that corroborate our claim are also discussed.

\section{RESULTS AND DISCUSSION}

1. Occurrence of the $\left[\mathrm{Fe}\left(\mathrm{H}_{2} \mathrm{O}\right)_{4}(\mathrm{NO})\right]^{2+}$ complex: Indications from AIMD simulations

An AIMD simulation of the brown ring complex in aqueous solution (aq) at ambient conditions were performed on $\mathrm{FeNOCl}_{2}$ solvated in $128 \mathrm{H}_{2} \mathrm{O}$ molecules in the canonical (NVT) ensemble using the Car-Parrinello algorithm within the CPMD package [18 20]. The 
simulations were carried out using the Becke-Perdew (BP86) pure DFT functional [21, 22] and an unrestricted wave function with quartet spin multiplicity within the local spin density approximation. The BP86 functional has been used earlier by Monsch and co-workers to study this complex [6]. The system was sampled for $40 \mathrm{ps}$ after an equilibration of $20 \mathrm{ps}$.

Further details of the AIMD simulation are provided in the $[\mathrm{IV}]$ section.

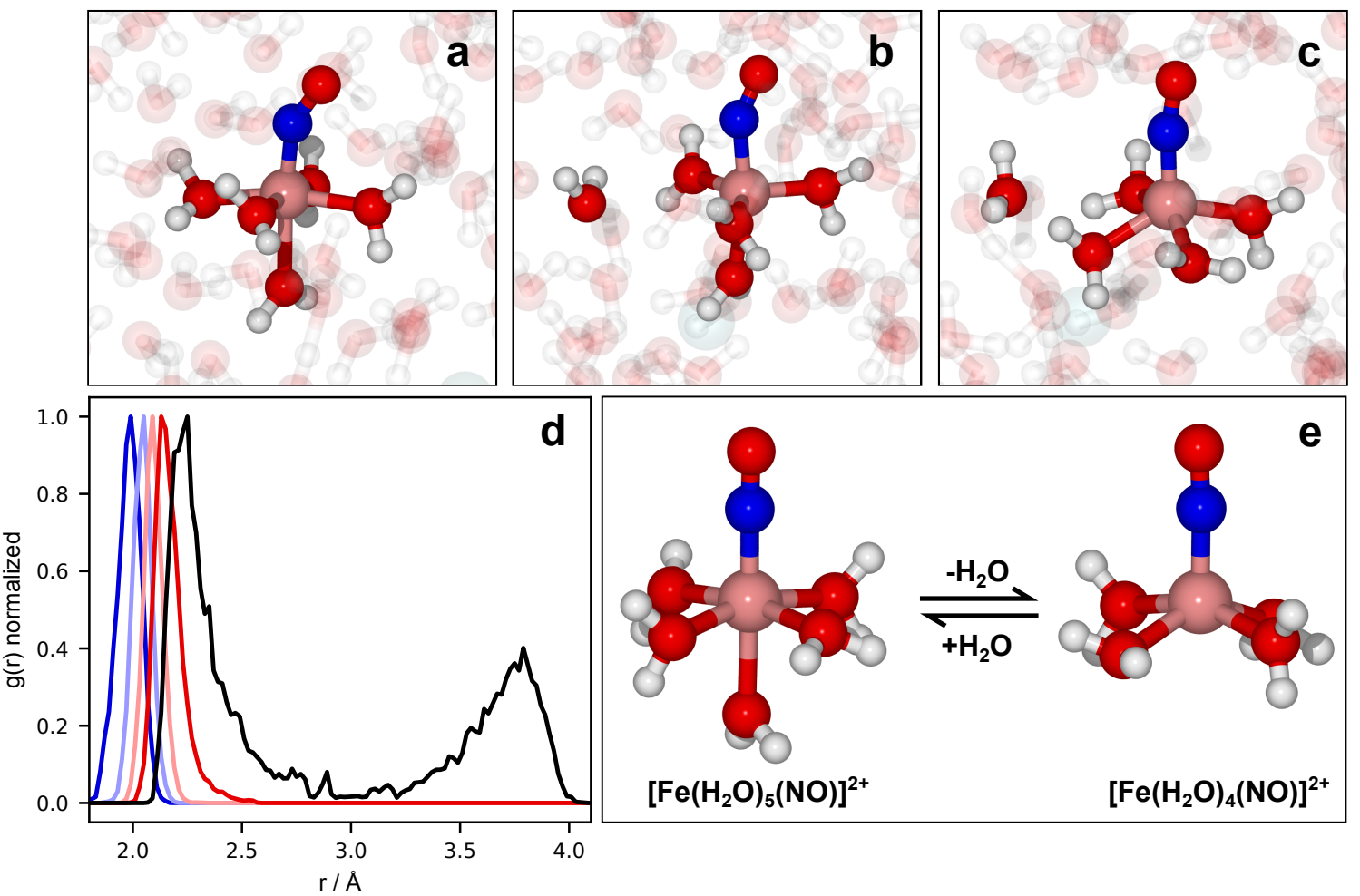

FIG. 1. Indication on the presence of $\left[\mathrm{Fe}\left(\mathrm{H}_{2} \mathrm{O}\right)_{4}(\mathrm{NO})\right]^{2+}$ from ab initio molecular dynamics simulations. a-c. Snapshots taken from the simulation showing the equilibrium between $\left[\mathrm{Fe}\left(\mathrm{H}_{2} \mathrm{O}\right)_{5}(\mathrm{NO})\right]^{2+}$ and $\left[\mathrm{Fe}\left(\mathrm{H}_{2} \mathrm{O}\right)_{5}(\mathrm{NO})\right]^{2+}$ through the exchange of one $\mathbf{H}_{2}$ O. Panel a shows the octahedral $\left[\mathrm{Fe}\left(\mathrm{H}_{2} \mathrm{O}\right)_{5}(\mathrm{NO})\right]^{2+}$ complex. The intermediate state with one equatorial $\mathrm{H}_{2} \mathrm{O}$ molecule dissociating from the penta-aqua complex is shown in panel $\mathbf{b}$. Panel c depicts the formation of the square-pyramidal $\left[\mathrm{Fe}\left(\mathrm{H}_{2} \mathrm{O}\right)_{4}(\mathrm{NO})\right]^{2+}$ complex. d. Radial distribution functions, normalized at the first peak to enhance features, for the five coordinating water molecule with respect to the Fe center. The black line shows the RDF for the Fe- $\mathrm{O}_{5}$ bond, showing its bimodal distribution and looseness, in stark contrast with the rest of the RDFs. In Figure S1, the corresponding curves with proper normalization is given. e. The optimized geometries of the $\left[\mathrm{Fe}\left(\mathrm{H}_{2} \mathrm{O}\right)_{5}(\mathrm{NO})\right]^{2+}$ and $\left[\mathrm{Fe}\left(\mathrm{H}_{2} \mathrm{O}\right)_{4}(\mathrm{NO})\right]^{2+}$ complex. 
Upon visualizing the trajectory obtained from the simulation, we found that the $\left[\mathrm{Fe}\left(\mathrm{H}_{2} \mathrm{O}\right)_{5}(\mathrm{NO})\right]^{2+}$ penta-aqua complex can lose an equatorial $\mathrm{H}_{2} \mathrm{O}$ molecule to form a $\left.\mathrm{Fe}\left(\mathrm{H}_{2} \mathrm{O}\right)_{4}(\mathrm{NO})\right]^{2+}$ tetraaqua species in which relaxation lead to a square-pyramidal geometry. $\left[\mathrm{Fe}\left(\mathrm{H}_{2} \mathrm{O}\right)_{5}(\mathrm{NO})\right]^{2+}$ can subsequently bind a water molecule from the bulk and revert back to the parent penta-aqua complex. The snapshots of these processes from the simulation are shown in Figure 1 1a-c. which clearly shows the phenomenon involving de-aquation and re-aquation. The simulation time is too short, though, in comparison to the time-scale of the processes to determine the statistics of this dynamical equilibrium. Also, as we show below the limitations of the BP86 functional approximation prohibit accurate free energy simulations to investigate the chemical equilibrium quantitatively. Instead, we focus on structural and electronic characteristics of the this duality of the brown ring complex.

In Figure 1 $1 \mathrm{~d}$, the radial distribution function (RDF) for each of the individual distances between the $\mathrm{Fe}^{2+}$ cation and the closest five water oxygen atoms $\left(\mathrm{O}_{i}\right.$ for $i=1-5$ at any given time) are presented.

An extra normalization to the peak maximum of each curve us used to enhance the differences in shape. In the supplementary information(SI), corresponding RDFs without the extra normalization are presented in Figure S1 and in Figure S2k-d in the SI, the RDF of the remaining water oxygen atoms relative to iron and the $\mathrm{Fe}-\mathrm{Cl} \mathrm{RDF}$ are plotted for comparison showing the second hydration shell and the outer sphere coordination of $\mathrm{Cl}^{-}$ anions. The iron nitrosyl RDFs are presented in Figure S2 $\mathrm{a}-\mathrm{b}$ in the SI.

In the first hydration shell of the brown ring complex, the four closest water molecules (RDFs of $\mathrm{Fe}-\mathrm{O}_{i}$ for $i=1-4$ ) are strongly bound to the iron cation, where as the fifth water molecule ( $\mathrm{RDF}$ of $\mathrm{Fe}-\mathrm{O}_{5}$ ) has a bimodal distribution signifying the occurrence of both penta-aqua and tetra-aqua complexes. Furthermore, the shape of the inner peak is much broader for $\mathrm{Fe}-\mathrm{O}_{5}$ than for $\mathrm{Fe}^{-} \mathrm{O}_{i}(i=1-4)$ reflecting a clearly softer iron water interaction of the fifth water molecule. Based on these simulation we propose that $\left[\mathrm{Fe}\left(\mathrm{H}_{2} \mathrm{O}\right)_{5}(\mathrm{NO})\right]^{2+}$ and $\left[\mathrm{Fe}\left(\mathrm{H}_{2} \mathrm{O}\right)_{4}(\mathrm{NO})\right]^{2+}$ species can co-exist in the aqueous solution.

It is very well know from previous studies that the $\left[\mathrm{Fe}\left(\mathrm{H}_{2} \mathrm{O}\right)_{5}(\mathrm{NO})\right]^{2+}$ species posses a multi-configurational wave function [6, 16]. Hence, the pure DFT description in the AIMD simulation is a limitation which prevents a quantitative analysis. However the simulations were performed within the unrestricted Kohn-Sham formalism which can to some extent capture the open shell character of the wave function as reflected by the presence of spin 
contamination. Hence, we performed static DFT computations with implicit solvation at the BP86/def2-TZVP/cpcm(water) level on both the penta-aqua and tetra-aqua complex and found the expectation value of the electron spin operator $\left\langle S^{2}\right\rangle$ to be 4.23 and 4.15 respectively, which clearly points to a substantial spin contamination. The BP86 functional has been shown to work well for this system, in previous studies, in reproducing molecular geometry and spin density in previous studies [6]. Thus, this interesting observation of the existence of a tetra-aqua complex along with the classically accepted penta-aqua complex, from the AIMD simulations, which has the aforementioned limitation, warrants a more careful investigation using more accurate static multi-configurational quantum chemical computations on static models.

\section{Multi-reference ab initio calculations and UV-vis spectra}

After observing the presence of $\left[\mathrm{Fe}\left(\mathrm{H}_{2} \mathrm{O}\right)_{4}(\mathrm{NO})\right]^{2+}$ and $\left[\mathrm{Fe}\left(\mathrm{H}_{2} \mathrm{O}\right)_{5}(\mathrm{NO})\right]^{2+}$ in the AIMD simulation in aqueous solution, the electronic structure and electronic excitations of the two complexes were studied thoroughly using multi-reference ab initio quantum chemical calculations in the ORCA program package [23]. Both these complexes have a quartet ground state. Geometries of the complexes were extracted from the MD simulation and optimized at the (U)TPSSh/def2-TZVP/cpcm(water) level of theory, which has been shown to function well in reproducing the geometry of Fe-NO complexes including the ones fraught with quasi-degeneracy [17, 24]. Thus we obtain the geometries in the absence of explicit solvating water molecules and its hydrogen bonding to the second solvation shell, but with implicit solvation i.e. cpcm(water), namely; (penta-aqua) $\left[\mathrm{Fe}\left(\mathrm{H}_{2} \mathrm{O}\right)_{5}(\mathrm{NO})\right]^{2+}$ and (tetraaqua) $\left[\mathrm{Fe}\left(\mathrm{H}_{2} \mathrm{O}\right)_{4}(\mathrm{NO})\right]^{2+}$. Nonetheless, we also decided to estimate the effects of the inclusion of explicit solvation. The interactions of the penta-aqua and tetra-aqua complexes with the surrounding solvent can only be accurately accounted for in a model including surrounding H-bonded water molecules. However, proper description of the electronic structure of the complexes on the other hand requires high-level multi-reference ab initio methods, like CASPT2/NEVPT2 [25, 26]. Consequently sampling of many different solvation structures is a challenge, and thus the geometry of a solvation model of the complex surrounded by 11 H-bonded water molecules was prepared and optimized at the same level of theory. It is important to note here that the models, both with and without explicit solvation, contain the 


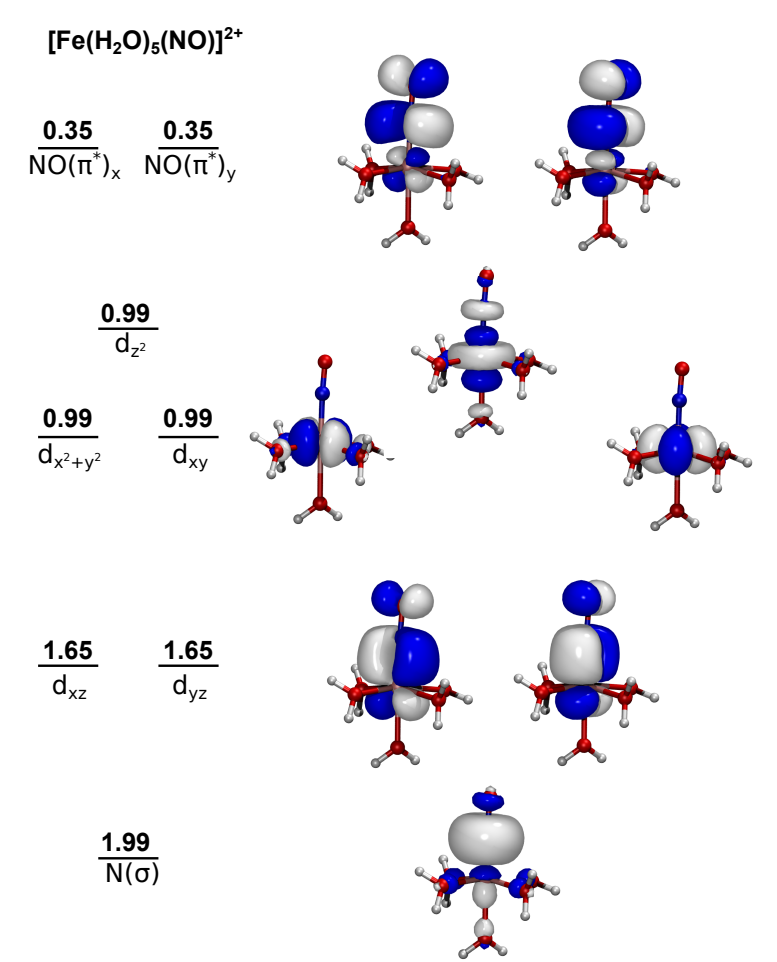

cpcm(water) implicit solvation in both geometry optimization and the electronic structure investigations with single point DFT and CASSCF/NEVPT2/CASPT2 calculations. From now on the model with 11 explicit solvating water molecules will be denoted as explicitly solvated complex, and if not mentioned otherwise the model with only implicit cpcm(water) solvation is indicated.

FIG. 2. Ground state molecular orbitals for the two relevant complexes. Orbitals obtained from state-specific $\operatorname{CASSCF}(9,8)$ computation for $\left[\mathrm{Fe}\left(\mathrm{H}_{2} \mathrm{O}\right)_{5}(\mathrm{NO})\right]^{2+}$ (left) and $\left[\mathrm{Fe}\left(\mathrm{H}_{2} \mathrm{O}\right)_{4}(\mathrm{NO})\right]^{2+}$ (right). The natural population is given above the orbitals in bold. The energies of the orbitals are mentioned below. The figure also describes the orbitals based on its character as mentioned alongside each orbital.

Considering geometries optimized at DFT level, we performed ab initio multi-reference computation on the penta-aqua and tetra-aqua complexes with and without explicit solvation. We employed a complete active space for the complexes with 9 electrons in 8 active orbitals, i.e. CAS $(9,8)$. The orbitals chosen for the active space constituted of the $\mathrm{N}(\sigma)$, the five $\mathrm{Fe}(3 \mathrm{~d})$ orbitals and the two $\mathrm{NO}\left(\pi^{*}\right)$ orbitals, included to accurately describe the electronic structure of the complex. This active space includes all the orbitals, from the valence orbital space, which account for the interaction between the Fe and N, of the NO moiety. 
NEVPT2 [26] (and CASPT2 [25]) calculations were performed on the same CAS $(9,8)$ reference wave function to account for dynamical correlation, which has been shown to play a central role in the description of electronic structure of Fe-NO complexes [15].

The brown ring complex has been established to be a $\{\mathrm{FeNO}\}^{7}$ species and hence doublet, quartet and sextet ground states are tentatively plausible. The doublet and sextet ground states are, however, significantly higher in energy as compared to the quartet ground state, see Table S1 in SI [27], for both penta-aqua and tetra-aqua complexes. Since we do not discuss physical/chemical processes where spin-orbit coupling is important in this study, we otherwise strictly confine our focus to the quartet manifold.
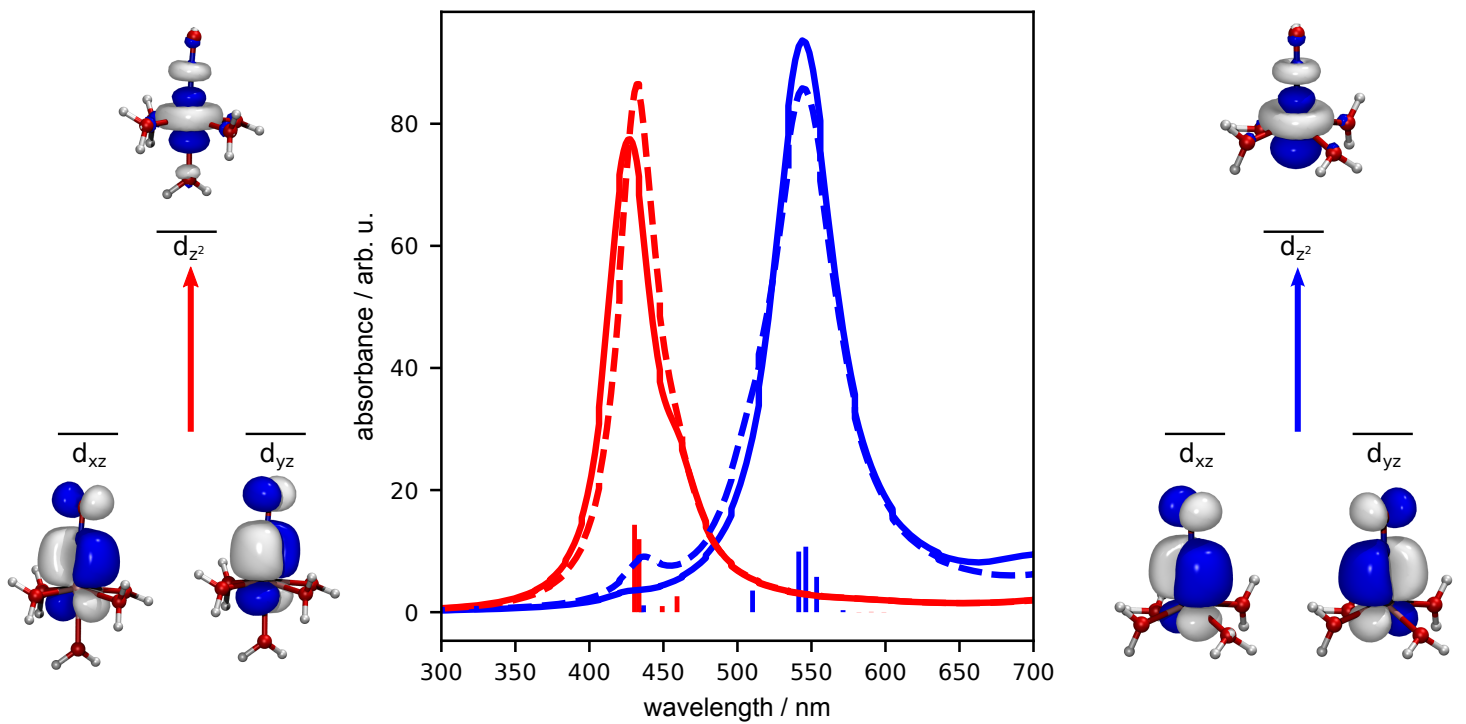

FIG. 3. Computed UV-vis spectra at the CASSCF $(9,8) / \operatorname{NEVPT2} /$ def2TZVP/cpcm(water) level of theory. Static spectra of the $\left[\mathrm{Fe}\left(\mathrm{H}_{2} \mathrm{O}\right)_{4}(\mathrm{NO})\right]^{2+}(\mathrm{red})$ and $\left[\mathrm{Fe}\left(\mathrm{H}_{2} \mathrm{O}\right)_{4}(\mathrm{NO})\right]^{2+}($ blue $)$ with(dotted line) and without(smooth line) explicit solvation. The orbitals involved in the most pronounced transitions are given alongside the spectra for $\left[\mathrm{Fe}\left(\mathrm{H}_{2} \mathrm{O}\right)_{5}(\mathrm{NO})\right]^{2+}($ left $)$ and $\left[\mathrm{Fe}\left(\mathrm{H}_{2} \mathrm{O}\right)_{4}(\mathrm{NO})\right]^{2+}($ right $)$.

The CASSCF/NEVPT2 calculations of the $\left[\mathrm{Fe}\left(\mathrm{H}_{2} \mathrm{O}\right)_{5}(\mathrm{NO})\right]^{2+}$ and $\left[\mathrm{Fe}\left(\mathrm{H}_{2} \mathrm{O}\right)_{4}(\mathrm{NO})\right]^{2+}$ complexes, as well as the explicitly solvated models, consistently show multi-reference character of the ground state wave function. The CASSCF optimized orbitals are shown in Figure 2. As clearly seen from Figure 2, the multi-reference nature arises due to the back-donation of electrons from the $\mathrm{Fe}\left(\mathrm{d}_{x z}\right.$ and $\left.\mathrm{d}_{y z}\right)$ orbitals to $\mathrm{NO}\left(\pi^{*}\right)$ orbital. This is reflected in the fractional natural occupation in these orbitals in agreement with earlier studies [6, 15, 17, 24]. 
Having determined the multi-reference wave function of the quartet ground state $\left(Q_{1}\right)$, consistent with previous computations, we investigate the quartet excited state manifold. We started with the computation of the classic brown ring complex, i.e. $\left[\mathrm{Fe}\left(\mathrm{H}_{2} \mathrm{O}\right)_{5}(\mathrm{NO})\right]^{2+}$. We performed a state averaged CASSCF, SA-CASSCF, over 10 quartet states with the same CAS $(9,8)$ active space, followed by NEVPT2. The energies of the excited states cover the relevant region in visible region, as indicated by UV spectrum given in Figure 1 of Wanat et al., and our calculated excitation energies are listed in Table S2 in SI [27]. Inclusion of more excited states in the State-Averaging can impact the quality of the CASSCF computation and moreover we are not interested in a excitation wavelength lower than 450-430 nm as per the focus of our study which is revealed later as the story unravels.

Upon plotting the the absorption spectra obtained at the NEVPT2 level, we found that the complex has a strong absorption at $430 \mathrm{~nm}$, see Figure 3. The same computation was also performed on the explicitly solvated model, with essentially the same spectra being produced. The color of a complex can easily be linked to its UV-vis spectra as is very well known in inorganic chemistry. According to this computation, the $\left[\mathrm{Fe}\left(\mathrm{H}_{2} \mathrm{O}\right)_{5}(\mathrm{NO})\right]^{2+}$ complex, with a the sharp absorption centered around $430 \mathrm{~nm}$, alone would give a yellow color to the solution. Moreover, the brown color, as seen in the brown ring test, is consistent with the UV-vis spectrum of the brown-ring system as measured by Wanat and co-workers [4] which contains two peak, one sharp peak at $450 \mathrm{~nm}$ and another weaker one at $585 \mathrm{~nm}$, as shown in Figure 1 in Wanat et al. [4]. The combination of strong absorption at 450 $\mathrm{nm}$ and weaker absorption at $585 \mathrm{~nm}$ can render the solution a brown color, but a lone peak at $450 \mathrm{~nm}$ cannot. Thus, according to the NEVPT2 calculations, the UV-vis spectrum of the penta-aqua species alone can not explain neither the color nor the dual peak in the experimental UV-vis spectra. Moreover the accurate reproduction of the peak at $450 \mathrm{~nm}$ by $\left[\mathrm{Fe}\left(\mathrm{H}_{2} \mathrm{O}\right)_{5}(\mathrm{NO})\right]^{2+}$ and absence of peak around $585 \mathrm{~nm}$, motivated us to further consider the possibility of a chemical equilibrium between a penta-aqua and a tetra-aqua species as indicated in the AIMD simulation.

Embarking on this idea we carried out the similar NEVPT2 calculations on 10 quartet states of the $\left[\mathrm{Fe}\left(\mathrm{H}_{2} \mathrm{O}\right)_{4}(\mathrm{NO})\right]^{2+}$ complex. In Figure 3, we clearly observe that the absorption for $\left[\mathrm{Fe}\left(\mathrm{H}_{2} \mathrm{O}\right)_{4}(\mathrm{NO})\right]^{2+}$ is centered around $550 \mathrm{~nm}$, a clear indication to the species behind the small long wavelength peak in the UV-vis spectra. Consequently the distinctive brown coloration in the brown ring test is henceforth ascribed not only due to the penta-aqua 
species, but also due to the presence of a tetra-aqua complex, $\left[\mathrm{Fe}\left(\mathrm{H}_{2} \mathrm{O}\right)_{4}(\mathrm{NO})\right]^{2+}$. The suggested possibility is new to the best of our knowledge and is a very interesting insight into a traditional chemical reaction, which we propose based on evidence provided with advanced quantum chemical methods including both static and dynamical correlation. It is worth mentioning here that the explicitly solvated model give essentially identical spectra as the one without, which signifies that the explicit solvation has a negligible effect on the electronic transitions in the spectra, according to this limited investigation of the solventsolute interactions. The excitation energies, obtained from NEVPT2 calculations, for both with and without explicitly solvated models were verified at the CASPT2 level of theory, with all the excitation energies found to be consistent with NEVPT2 within 0.3-0.4 eV variation, see Table S2 and S3 in SI [27].

The nature of the electronic transitions, involved in the relevant peaks discussed above, were deduced by inspecting the natural population and natural orbitals of the respective excited states. For $\left[\mathrm{Fe}\left(\mathrm{H}_{2} \mathrm{O}\right)_{5}(\mathrm{NO})\right]^{2+}$ the peak at $430 \mathrm{~nm}(\mathrm{NEVPT} 2)$ primarily arises as a result of transitions to the $\mathrm{Q}_{9}$ and $\mathrm{Q}_{10}$ states. These two states are $\mathrm{d}-\mathrm{d}$ transition states, generated by transitions of an electron from the $\mathrm{d}_{x z}$ and $\mathrm{d}_{y z}$ orbitals to the $\mathrm{d}_{z^{2}}$ orbital, see Figure 3. Not having center of symmetry d-d transitions for these complexes can give rise to and in fact results in bright transitions. The peak at $550 \mathrm{~nm}$ for $\left[\mathrm{Fe}\left(\mathrm{H}_{2} \mathrm{O}\right)_{4}(\mathrm{NO})\right]^{2+}$ results due to transitions to the $\mathrm{Q}_{7}$ and $\mathrm{Q}_{8}$ states, which correspond to the same $\mathrm{d}-\mathrm{d}$ transition states as seen for the penta-aqua complex, involving the same set of Fe-d orbitals. The removal of an $\mathrm{H}_{2} \mathrm{O}$ stabilizes the $\mathrm{d}$-d transition for the tetra-aqua species more than in the penta-aqua species. Hence, the $\mathrm{Q}_{9}$ and $\mathrm{Q}_{10}$ states in the penta-aqua complex correspond to the $\mathrm{Q}_{7}, \mathrm{Q}_{8}$ states in the tetra-aqua complex and the peak for the penta-aqua complex is red shifted in the tetra-aqua complex. The $\mathrm{d}_{z^{2}}$ orbital which is axially directed, for both these two complexes, accepts the excited electron. The removal of the water molecule reduces the electron density along the axial direction. This in turn reduces the Coulomb repulsion experienced by the excited electron in the $d_{z^{2}}$ orbital, thus stabilizing these two states, and hence red shifting the peak. The lower excited states are of metal-to-ligand charge-transfer (MLCT) in nature with transition from the metal center to the $\mathrm{NO}\left(\pi^{*}\right)$, followed by the transfer of other metal centered $\mathrm{d}$-d transitions which corresponds to transition from $\mathrm{d}_{x z}$, $\mathrm{d}_{y z}$ to $\mathrm{d}_{x y}$ and $\mathrm{d}_{x^{2}+y^{2}}$. The ordering of these metal centered (MC) states are changed as one goes from penta-aqua to tetra-aqua species along with the shift in the peak position. 
Motivated by the static computations and the strong indication that the UV-vis spectra cannot be explained by a single complex, we proceeded towards a more accurate simulation of the UV-vis spectra of the penta-aqua and tetra-aqua species giving more realism in the comparison to the experimental spectrum. Not much improvement can be done on the electronic structure front as we are already employing the highly accurate NEVPT2 method, and verified them by CASPT2 calculations. Instead we resorted to spectrum simulations at the NEVPT2 level of theory with Wigner sampling of geometries at DFT level. 101 geometries of both the complexes were sampled using temperature dependent Wigner distribution, at $293 \mathrm{~K}$, as implemented in SHARC-2.1.1, [28]. Temperature dependent Wigner distribution has been recently shown to accurately produce the UV-vis absorption spectra of transition metals complexes [29]. The temperature dependent Wigner distribution is essential in reproducing the spectra if floppy vibrations are present. The Wigner sampled $\mathrm{UV}$-vis spectrum for $\left[\mathrm{Fe}\left(\mathrm{H}_{2} \mathrm{O}\right)_{5}(\mathrm{NO})\right]^{2+}$ clearly shows the peak at $440 \mathrm{~nm}$, which practically coincides with the sharp peak at $450 \mathrm{~nm}$ in the experimental spectra, see Figure 4. The simulated spectrum for $\left[\mathrm{Fe}\left(\mathrm{H}_{2} \mathrm{O}\right)_{4}(\mathrm{NO})\right]^{2+}$ at the same level of theory shows a peak, of much diminished intensity as compared to the one at $440 \mathrm{~nm}$, at around $580 \mathrm{~nm}$, which is clearly identified with the peak at $590 \mathrm{~nm}$ in the experimental UV-vis spectrum. This close reproduction of the UV-vis spectrum at the temperature dependent Wigner sampled spectra, at the NEVPT2 level of theory, enhances the credibility of our hypothesis.

Having established that the UV-vis spectrum and hence the brown color of the brown ring cannot be solely explained by a single complex, we turn our attention towards the energetics of the two species, whose existence are indicated by the AIMD simulation. Due to the multireference nature of the electronic ground state in both the complexes, the pure functional with unrestricted wave function, though can accurately predict the geometries, in such case, are often unable to accurately estimate the relative energetics between the complexes. Hence we also compared the energies of the complexes at the CASSCF $(9,8) / \mathrm{NEVPT} 2$ level of theory. The release of an $\mathrm{H}_{2} \mathrm{O}$ from $\left[\mathrm{Fe}\left(\mathrm{H}_{2} \mathrm{O}\right)_{5}(\mathrm{NO})\right]^{2+}$ to give $\left[\mathrm{Fe}\left(\mathrm{H}_{2} \mathrm{O}\right)_{4}(\mathrm{NO})\right]^{2+}$ brings about two important changes in the system, which are opposing to each other in enthalpic terms. There is a loss of $\mathrm{Fe}-\mathrm{OH}_{2}$ bond and the gain of two extra H-bonds, that the evolved $\mathrm{H}_{2} \mathrm{O}$ can form with its two lone pairs. In the aqueous solutions there are of course numerous hydrogen bonding opportunities, but we believe that the limited models may capture the essential physics in the process. 


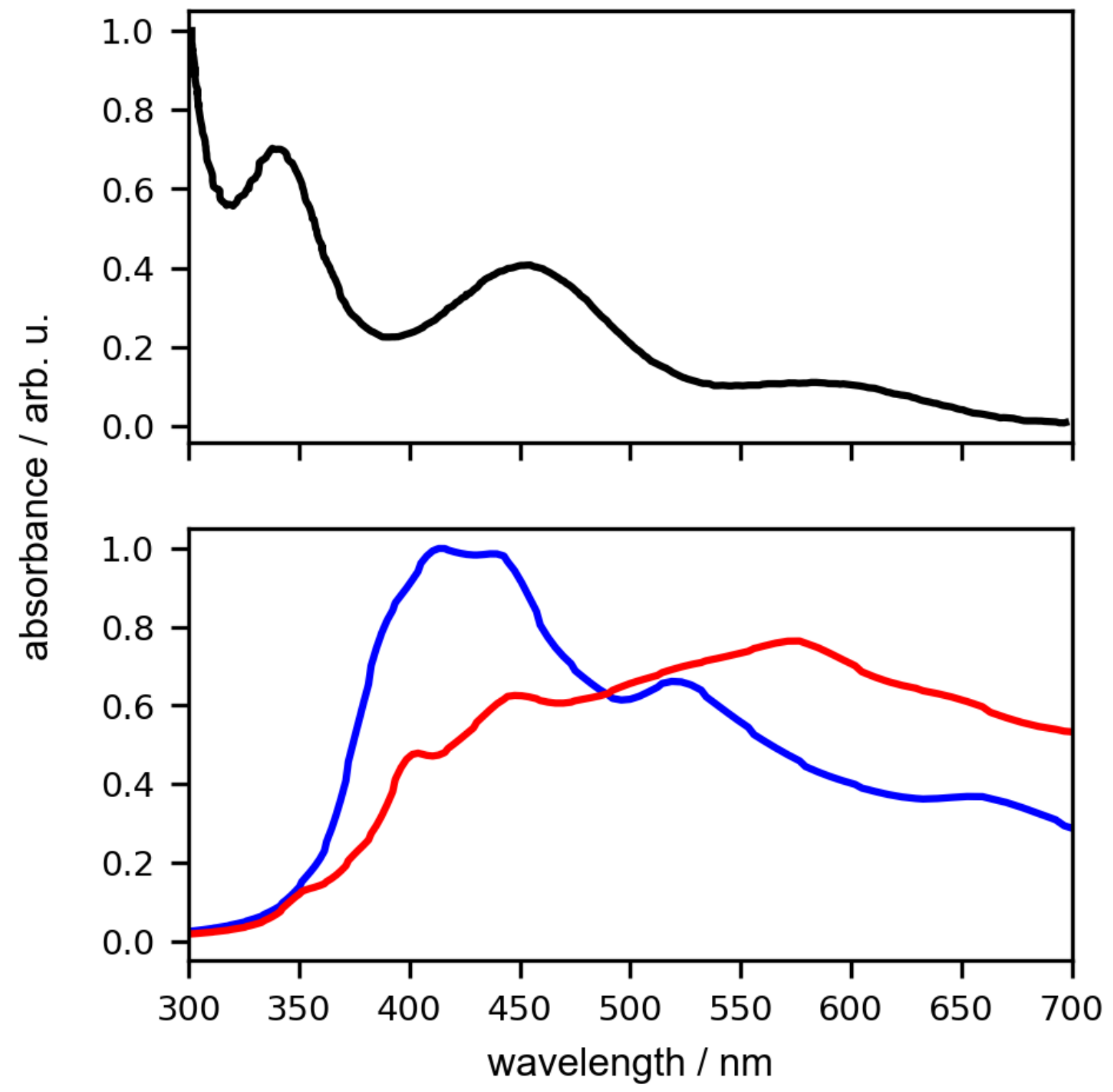

FIG. 4. Simulated UV-vis spectra based on a temperature dependent Wigner distribution. Spectra for $\left[\mathrm{Fe}\left(\mathrm{H}_{2} \mathrm{O}\right)_{5}(\mathrm{NO})\right]^{2+}(\mathrm{red})$ and $\left[\mathrm{Fe}\left(\mathrm{H}_{2} \mathrm{O}\right)_{4}(\mathrm{NO})\right]^{2+}($ blue $)$ at CASSCF $(9,8) / \mathrm{NEVPT} 2 / \mathrm{TZVP} / \mathrm{cpcm}$ (water) level of theory averaged over 101 geometries sampled from the temperature dependent Wigner distribution at $293 \mathrm{~K}$. The black line in the top panel represents the experimental UV-vis spectra as extracted and reproduced from Figure 1 in Wanat et al. [4].

Energetically apart from the free energy gain when a water dissociates, the bond dissociation energy, of $\mathrm{Fe}-\mathrm{OH}_{2}$ bond, can partly be compensated by the gain of H-bonds that 

computation.

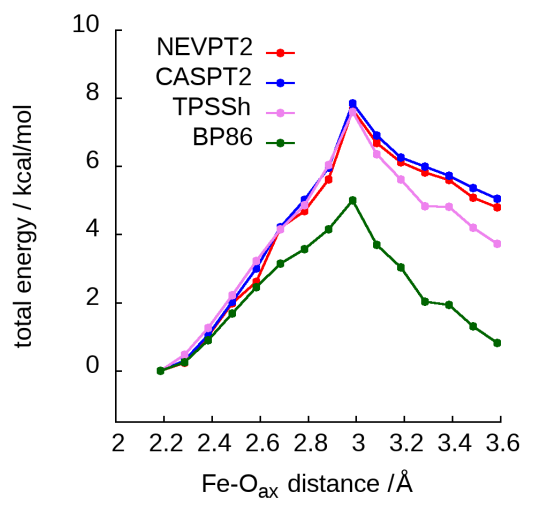

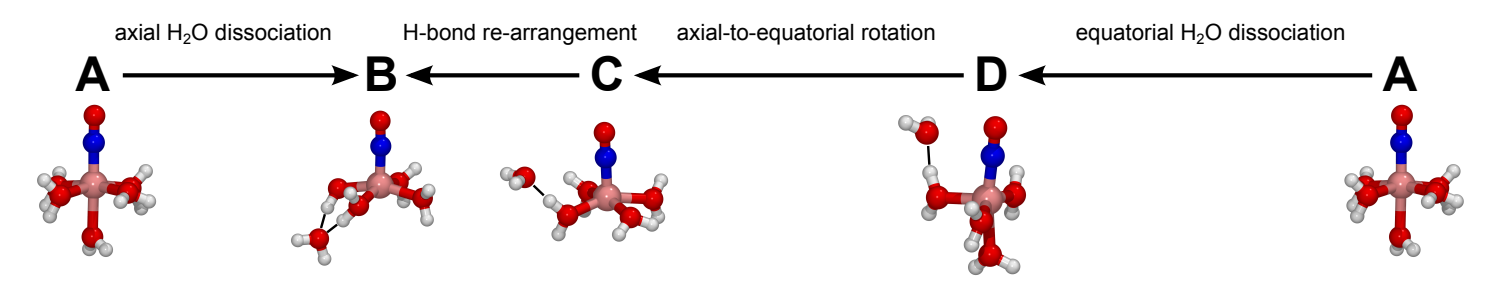

the released water molecule forms. As the system is inherently multi-reference, we can a priori only trust energetics obtained at CASSCF/NEVPT2 level of theory. We performed a relaxed scan along the reaction coordinates, as identified from ab-initio MD simulation, at the TPSSh/def-TZVP/cpcm(water) level of theory, by relaxing all the internal coordinates other than the fixed scanned coordinate at each point. Following this, these geometries were used for CASSCF/NEVPT2/ZORA-def2-TZVP and CASSCF/CASPT2/ZORA-def2-TZVP single point computations along the scans. Please see IV] section for details on energetics

FIG. 5. Cuts in the ground state potential energy surface along particular reaction coordinates for different quantum chemical methods. Potentials for NEVPT2(black), CASPT2(blue), TPSSh(violet) and BP86(green) for the relaxed scan along the different reaction coordinate. From left to right we show the scans along $\mathrm{Fe}-\mathrm{O}_{a x} . \mathrm{O}_{e q}^{\prime}-\mathrm{Fe}-\mathrm{O}_{a x}$ bond angle and Fe- $\mathrm{O}_{e q}$ degree of freedom, respectively. The H-bonding formed by the $\mathrm{H}_{2} \mathrm{O}$ dissociation is shown with the dotted black lines in the figure in the right. Two pathways and the corresponding reaction coordinates are depicted for transformation from $\mathbf{A}$ to $\mathbf{B}$ and is highlighted at the top of the figure. The process representing that particular reaction coordinate is also mentioned above the corresponding arrows.

As identified from the AIMD simulation and highlighted in Figure 1a-c, we see that firstly the equatorial $\mathrm{H}_{2} \mathrm{O}$ dissociates from the penta-aqua complex. This leads to the formation of a distorted trigonal pyramidal tetra-aqua complex (see Figure 1 $\mathrm{b}$ ) which functions as 
an intermediate state. Subsequently the axial $\mathrm{H}_{2} \mathrm{O}$ increases the $\mathrm{O}_{e q}^{\prime}-\mathrm{Fe}-\mathrm{O}_{a x}$ angle in the formation of a square-pyramidal complex, see Figure 1 1 . In Figure 5 we plot cuts in the ground state potential energy surface, described at the DFT, NEVPT2 and CASPT2 levels of theory, along relevant reaction coordinates.

We looked into the possibility of both equatorial as well as axial $\mathrm{H}_{2} \mathrm{O}$ dissociation, by performing relaxed scans along the corresponding $\mathrm{Fe}-\mathrm{OH}_{2}$ bond distances. Additionally we also performed a relaxed scan along the $\mathrm{O}_{e q}^{\prime}-\mathrm{Fe}-\mathrm{O}_{a x}$ bond angle following the last point in the equatorial $\mathrm{H}_{2} \mathrm{O}$ dissociation scan, where the $\mathrm{O}_{e q}^{\prime}$ denotes the oxygen atom in the water molecule directly opposite to the one(equatorial, i.e. $\mathrm{O}_{e q}$ ) which dissociates.

We denote different species on the ground state potential energy surface by alphabets. The dissociation of an equatorial $\mathrm{H}_{2} \mathrm{O}$ from the octahedral penta-aqua complex (A) gives rise to a distorted trigonal-bi-pyramidal tetra-aqua complex $(\mathbf{D})$ which then undergoes a relaxation along the $\mathrm{O}_{e q}^{\prime}-\mathrm{Fe}-\mathrm{O}_{a x}$ bond angle to give the square-pyramidal complex $(\mathbf{C})$. This then forms an additional $\mathrm{H}$ bond and relaxes to the lowest possible configuration for the tetra-aqua complex $(\mathbf{B})$. So this forms a three step process from $\mathbf{A}$ to $\mathbf{B}$. This is more or less also the pathway shown by AIMD. However if we do an axial $\mathrm{H}_{2} \mathrm{O}$ dissociation scan we found that $\mathbf{A}$ directly transforms to $\mathbf{B}$ and that too with a lower energy barrier with respect to the the three step process discussed earlier. This indicates the axial dissociation to be the plausibly predominant pathway.

Comparing the performance of different quantum chemical approximations, the NEVPT2 and CASPT2 potential energy surfaces are practically identical with sub kcal/mol difference, whereas DFT predicts a more stable tetra-aqua species, as clearly seen in Figure 5. The extra stabilization for the tetra-aqua species, produced by BP86 is a clear deviation from more accurate NEVPT2 and CASPT2. This corroborates our claim that it is wise to avoid energy estimations from the CPMD simulations and alternate routes are discussed later. The inclusion of exact exchange and higher order kinetic energy terms in the meta-hybrid TPSSh functional yields a close reproduction of NEVPT2 and CASPT2 results, which justifies our geometry optimizations with TPSSh. It also opens up a latter avenue of performing AIMD with this particular functional. In addition we observe, as seen in both the $\mathrm{Fe}-\mathrm{O}_{a x}$ and Fe- $\mathrm{O}_{e q}$ bond distance scans from Figure 5, that the energy steadily increases as the Fe-O bond is broken, reaching a maxima, where the released water molecule forms two H-bonds 
with the equatorial water molecules, as shown by black lines in Figure 5 . The last step of the scan is seen to be only $\sim 4.7 \mathrm{kcal} / \mathrm{mol}$ in energy above the initial point, i.e. the penta-aqua complex. Consequently the formation of the $\left[\mathrm{Fe}\left(\mathrm{H}_{2} \mathrm{O}\right)_{4}(\mathrm{NO})\right]^{2+}$ is energetically stabilized by the formation of two extra H-bonding, as highlighted by black lines in Figure 5

There is also the gain in the free energy which we have not considered in these cluster models yet. Now the computation of the free energy of dissociation in the solvent phase is particularly difficult in this system, since the failure in energetics of the DFT framework does not allow for sampling of the free energy difference in the AIMD framework. We have tried to address this in a way which is commonly practiced and well established when computing the free energy profile of reaction mechanisms [30]. We have optimized the penta-aqua, tetra-aqua and a water molecule separately at (U)TPSSh/def2-TZVP/cpcm(water) level of theory, followed a frequency computation. The free energy correction were obtained, which were scaled by a factor of 0.5 which has been shown to be standard protocol by many early studies [30, 31]. The free energy correction, obtained by the aforementioned protocol, for the reaction, $\left[\mathrm{Fe}\left(\mathrm{H}_{2} \mathrm{O}\right)_{5}(\mathrm{NO})\right]^{2+} \rightarrow\left[\mathrm{Fe}\left(\mathrm{H}_{2} \mathrm{O}\right)_{4}(\mathrm{NO})\right]^{2+}+\mathrm{H}_{2} \mathrm{O}$, turns out to be $-4.9 \mathrm{kcal} / \mathrm{mol}$. Thus the total free energy change for the water dissociation process can be approximated to be very near to the $\sim 0 \mathrm{kcal} / \mathrm{mol}[\sim(4.7-4.9) \mathrm{kcal} / \mathrm{mol}]$. Accurate estimation would require a much more detailed study like the one performed by Dixon and co-workers, and Spencer and co-workers for uranium and plutonium complexes [32 34, and so only an approximate value is mentioned. Moreover we computed the energy difference between the penta-aqua and tetra-aqua complex with the explicitly solvated model. For the explicitly solvated model, for which we were able to optimize two different H-bonding configurations, the energy for the $\left[\mathrm{Fe}\left(\mathrm{H}_{2} \mathrm{O}\right)_{4}(\mathrm{NO})\right]^{2+}$ was found to be 1.36 and $5.5 \mathrm{kcal} / \mathrm{mol}$ above $\left[\mathrm{Fe}\left(\mathrm{H}_{2} \mathrm{O}\right)_{5}(\mathrm{NO})\right]^{2+}$, with the lower value predicted when two extra H-bonds are present. The energetic profiling clearly shows that fluctuations in the H-bonding environment around the solute can favor one species over the other. Thus based of NEVPT2 and CASPT2 energetics which are considered as gold standards in ab initio multi-reference PT level of theories, the occurrence of both these complex in the aqueous solution observed in the AIMD simulations is plausible. 


\section{CONCLUSION}

This study has revealed a new aspect of the well known brown ring test. Our AIMD simulations and multi-reference ab initio perturbation theories give strong indications of the existence of the $\left[\mathrm{Fe}\left(\mathrm{H}_{2} \mathrm{O}\right)_{4}(\mathrm{NO})\right]^{2+}$ complex in the solution along with the commonly considered $\left[\mathrm{Fe}\left(\mathrm{H}_{2} \mathrm{O}\right)_{5}(\mathrm{NO})\right]^{2+}$. We also establish based on SA-CASSCF/NEVPT2 computation that $\left[\mathrm{Fe}\left(\mathrm{H}_{2} \mathrm{O}\right)_{5}(\mathrm{NO})\right]^{2+}$ alone cannot impart the brown coloration to the brown-ring test. Instead it is the result of the presence of both $\left[\mathrm{Fe}\left(\mathrm{H}_{2} \mathrm{O}\right)_{5}(\mathrm{NO})\right]^{2+}$ and $\left[\mathrm{Fe}\left(\mathrm{H}_{2} \mathrm{O}\right)_{4}(\mathrm{NO})\right]^{2+}$, which adds the dimension of dynamic complexity to this case of text book chemistry.

It is difficult to experimentally verify the existence of the two species in solution due to the inherent instability of the system, but it may be possible with advanced spectroscopic techniques. However, sample delivery is challenge due to necessity of dissolution of NO and the instability of the complex itself. Core-level spectroscopy at the iron edge would be expected to show a bimodal signal in the presence of both the penta-aqua and tetra-aqua species. We also propose that $\left[\mathrm{Fe}\left(\mathrm{H}_{2} \mathrm{O}\right)_{5}(\mathrm{NO})\right]^{2+}$ and $\left[\mathrm{Fe}\left(\mathrm{H}_{2} \mathrm{O}\right)_{4}(\mathrm{NO})\right]^{2+}$ species will have strikingly different extended x-ray absorption fine structure (EXAFS) signature. Mössbauer spectroscopy could be of help and thus we computed the Mössbauer splitting for both the penta-aqua and tetra-aqua species, at CASSCF level of theory for the explicitly solvated complexes, and found the values to be $2.1 \mathrm{~mm} / \mathrm{s}$ and $1.2 \mathrm{~mm} / \mathrm{s}$ respectively. Additionally change of solvent could be done judiciously to favor one species over the other and then individual UV-vis or other spectra could be obtained.

Our serendipitous finding and the bold suggestion, that follows from it, challenges a well known notion about an even better known reaction. In consequence, this study opens up new challenges for our experimental colleagues to verify or refute this suggestion using the very state-of-the-art spectroscopic techniques, though as our theory is based on sound electronic structure methods we strongly believe the two species to play an important role in the chemistry of the brown ring test. 


\section{METHODS}

\section{A. Ab initio molecular dynamics simulations}

The AIMD simulation was performed of a system containing altogether $\mathrm{Fe}(\mathrm{NO}) \mathrm{Cl}_{2}$ and 128 water molecules at $300 \mathrm{~K}$ using the Nosé-Hoover [35, 36] chain thermostat for sampling within the NVT ensemble. In the Car-Parrinello algorithm, we used a fictitious mass of 500 a.u. for the electronic orbital degrees of freedom and a time step of 0.072 fs for the integration of the equations of motion. The Packmol package [37] was used to pack 123 water molecules along with $\left[\mathrm{Fe}\left(\mathrm{H}_{2} \mathrm{O}\right)_{5}(\mathrm{NO})\right]^{2+}$ and two $\mathrm{Cl}^{-}$anions in a cubic box of size $15.9911 \AA$ to obtain a density of $0.9999 \mathrm{~g} / \mathrm{cm}^{3}$ (or actually $1.1046 \mathrm{~g} / \mathrm{cm}^{3}$ since the system was deuterated to reduce the spectral overlap between nuclear motion and fictitious electron degrees of freedom). The system was equilibrated for $20 \mathrm{ps,} \mathrm{confirmed} \mathrm{by} \mathrm{the} \mathrm{subsequent}$ absence of drift in potential energy. The fictitious velocities was reset to zero every 10 ps to avoid heating up of the electronic degrees of freedoms. The trajectory was sampled every 20 time step.

A plane wave basis set with a cut-off of 70 Ry was used and the core electrons were treated with Troullier-Martins [38 norm-conserving pseudo-potentials expressed in the KleinmanBylander form [39]. Pseudo-potentials for nitrogen, oxygen, and chlorine included $\mathrm{p}$ and s channels and were non-local in the s channel, whereas for that of hydrogen only had an s channel. For iron, non-linear exchange and correlation core-corrections [40] were employ with a pseudo-potential containing $\mathrm{d}$ and s channels and non-local in the d channel.

\section{B. Quantum chemical computation}

Implicit solvation with water as solvent was accounted by $\mathrm{cpcm}$ model for all quantum chemical computations. All DFT optimization, scan and frequency computations were done with the Gaussian 16 quantum chemical package [41]. The free energy correction was done following the protocol described in references 30 , 31 using the thermal corrections given by frequency calculations. The free energy correction $\mathrm{G}_{\text {corr }}$ and $\mathrm{H}_{\text {corr }}$ was obtained from the frequency computations. Though the free energy corrections were computed using cpcm(water) solvation, Sackur-Tetrode equation which follows the ideal gas approximation was used. For this reason the entropy is scaled by factor 0.5 when computing free energy 
changes in condensed phase.

$$
\begin{gathered}
-T S_{\text {sol }}=0.5\left[G_{\text {corr }}-H_{\text {corr }}\right] \\
G_{\text {sol }}=E_{\text {sol }}+H_{\text {corr }}+0.5\left[G_{\text {corr }}-H_{\text {corr }}\right]
\end{gathered}
$$

\section{ACKNOWLEDGMENTS}

M.O. acknowledges funding from the European Union's Horizon 2020 research and innovation programme under the Marie Skłodowska-Curie grant agreement No 860553. A.B. and M.O. acknowledge funding from the Carl Tryggers Foundation (contract CTS18:285). The calculations were partially enabled by resources provided by the Swedish National Infrastructure for Computing (SNIC) at the Swedish National Supercomputer Center (NSC), the High Performance Computer Center North (HPC2N), and Chalmers Centre for Computational Science and Engineering (C3SE) partially funded by the Swedish Research Council through grant agreement no. 2018-05973.

Competing Interests The authors declare no competing financial or non-financial interests.

Author contributions The project was designed and led by A.B and M.O. Simulations were performed and analyzed by A.B. and M.C. The manuscript was written by A.B., M.C., and M.O. 
Correspondence Correspondence and requests for materials should be addressed to A.B. and M.O.

[1] Manchot, W. \& Zechentmayer, K. Ueber die ferroverbindungen des stickoxydes. Justus Liebigs Annalen der Chemie 350, 368-389 (1906).

[2] Manchot, W. \& Huttner, F. Ueber die ferroverbindungen des stickoxydes. Justus Liebigs Annalen der Chemie 372, 153-178 (1910).

[3] Manchot, W. Demonstrationsversuche mit ferrostickoxyd-verbindungen. Berichte der deutschen chemischen Gesellschaft 47, 1614-1616 (1914).

[4] Wanat, A. et al. Kinetics, mechanism, and spectroscopy of the reversible binding of nitric oxide to aquated Iron (II). an undergraduate text book reaction revisited. Inorganic Chemistry 41, 4-10 (2002).

[5] Enemark, J. \& Feltham, R. Principles of structure, bonding, and reactivity for metal nitrosyl complexes. Coordination Chemistry Reviews 13, 339-406 (1974).

[6] Monsch, G. \& Klüfers, P. $\left[\mathrm{Fe}(\mathrm{H} 2 \mathrm{O})_{5}(\mathrm{NO})\right]^{2+}$, the "brown-ring" chromophore. Angewandte Chemie International Edition 58, 8566-8571 (2019).

[7] Thomas, D. J. \& Lehnert, N. The Biocoordination Chemistry of Nitric Oxide With Heme and Nonheme Iron Centers (Elsevier, 2017).

[8] Butler, A. R. \& Megson, I. L. Non-heme iron nitrosyls in biology. Chemical Reviews 102, 1155-1166 (2002).

[9] Chakraborty, S. et al. Spectroscopic and computational study of a nonheme iron nitrosyl center in a biosynthetic model of nitric oxide reductase. Angewandte Chemie International Edition 53, 2417-2421 (2014).

[10] Griffith, W., Lewis, J. \& Wilkinson, G. Some nitric oxide complexes of iron and copper. Journal of the Chemical Society (Resumed) 3993-3998 (1958).

[11] Conradie, J., Hopmann, K. H. \& Ghosh, A. Understanding the unusually straight: a search for MO insights into linear $\{\mathrm{FeNO}\}^{7}$ units. The Journal of Physical Chemistry B 114, 8517-8524 (2010).

[12] Cheng, H.-Y., Chang, S. \& Tsai, P.-Y. On the "brown-ring" reaction product via densityfunctional theory. The Journal of Physical Chemistry A 108, 358-361 (2004). 
[13] Burkhardt, L. et al. Electronic structure of the hieber anion $\left[\mathrm{Fe}(\mathrm{CO})_{3}(\mathrm{NO})\right]^{-}$revisited by x-ray emission and absorption spectroscopy. Inorganic Chemistry 59, 3551-3561 (2020).

[14] Klein, J. E. et al. The electronic ground state of $\left[\mathrm{Fe}(\mathrm{CO})_{3}(\mathrm{NO})\right]^{-}$: a spectroscopic and theoretical study. Angewandte Chemie International Edition 53, 1790-1794 (2014).

[15] Radon, M., Broclawik, E. \& Pierloot, K. Electronic structure of selected $\{\mathrm{FeNO}\}^{7}$ complexes in heme and non-heme architectures: A density functional and multireference ab initio study. The Journal of Physical Chemistry B 114, 1518-1528 (2010).

[16] Pierloot, K., Phung, Q. M. \& Ghosh, A. Electronic structure of neutral and anionic ironnitrosyl corrole. a multiconfigurational and density matrix renormalization group investigation. Inorganic Chemistry 59, 11493-11502 (2020).

[17] Banerjee, A., Sen, S. \& Paul, A. Theoretical investigations on the mechanistic aspects of $\mathrm{O}_{2}$ activation by a biomimetic Dinitrosyl Iron Complex. Chemistry-A European Journal 24, 3330-3339 (2018).

[18] Andreoni, W. \& Curioni, A. New advances in chemistry and material science with cpmd and parallel computing. Parallel Computing 26, 819-842 (2000).

[19] http://www.cpmd.org/. Copyright ibm corp 1990-2008. Copyright MPI fur Festkorperforschung Stuttgart 1997-2001.

[20] Car, R. \& Parrinello, M. Unified approach for molecular dynamics and density-functional theory. Physical Review Letters 55, 2471 (1985).

[21] Becke, A. D. Density-functional exchange-energy approximation with correct asymptotic behavior. Physical Review A 38, 3098 (1988).

[22] Perdew, J. P. Density-functional approximation for the correlation energy of the inhomogeneous electron gas. Physical Review B 33, 8822 (1986).

[23] Neese, F., Wennmohs, F., Becker, U. \& Riplinger, C. The ORCA quantum chemistry program package. The Journal of Chemical Physics 152, 224108 (2020).

[24] Ye, S. \& Neese, F. The unusual electronic structure of dinitrosyl iron complexes. Journal of the American Chemical Society 132, 3646-3647 (2010).

[25] Finley, J., Malmqvist, P.-A., Roos, B. O. \& Serrano-Andrés, L. The multi-state CASPT2 method. Chemical Physics Letters 288, 299-306 (1998).

[26] Angeli, C., Cimiraglia, R., Evangelisti, S., Leininger, T. \& Malrieu, J.-P. Introduction of n-electron valence states for multireference perturbation theory. The Journal of Chemical 
Physics 114, 10252-10264 (2001).

[27] Supplementary information available at.... (2021).

[28] Mai, S., Marquetand, P. \& González, L. Nonadiabatic dynamics: The sharc approach. Wiley Interdisciplinary Reviews: Computational Molecular Science 8, e1370 (2018).

[29] Wernbacher, A. M. \& Gonzalez, L. The importance of finite temperature and vibrational sampling in the absorption spectrum of a nitro-functionalized $\mathrm{Ru}(\mathrm{II})$ water oxidation catalyst. Physical Chemistry Chemical Physics (2021).

[30] Spickermann, C. First Principles Approaches to the Entropy of Condensed Phases and Complex Systems. Springer Theses (Springer International Publishing, 2010).

[31] Wertz, D. H. Relationship between the gas-phase entropies of molecules and their entropies of solvation in water and 1-octanol. Journal of the American Chemical Society 102, 5316-5322 (1980).

[32] Gutowski, K. E. \& Dixon, D. A. Predicting the energy of the water exchange reaction and free energy of solvation for the uranyl ion in aqueous solution. The Journal of Physical Chemistry A 110, 8840-8856 (2006).

[33] Odoh, S. O., Bylaska, E. J. \& De Jong, W. A. Coordination and hydrolysis of plutonium ions in aqueous solution using Car-Parrinello molecular dynamics free energy simulations. The Journal of Physical Chemistry A 117, 12256-12267 (2013).

[34] Spencer, S. et al. Hydration of $\mathrm{UO}_{2}{ }^{2+}$ and $\mathrm{PuO}_{2}{ }^{2+}$. The Journal of Physical Chemistry A 103, 1831-1837 (1999).

[35] Nosé, S. A unified formulation of the constant temperature molecular dynamics methods. The Journal of Chemical Physics 81, 511-519 (1984).

[36] Hoover, W. G. Canonical dynamics: Equilibrium phase-space distributions. Physical Review A 31, 1695 (1985).

[37] Martínez, L., Andrade, R., Birgin, E. G. \& Martínez, J. M. Packmol: a package for building initial configurations for molecular dynamics simulations. Journal of Computational Chemistry 30, 2157-2164 (2009).

[38] Troullier, N. \& Martins, J. L. Efficient pseudopotentials for plane-wave calculations. Phys. Rev. B 43, 1993-2006 (1991).

[39] Kleinman, L. \& Bylander, D. M. Efficacious form for model pseudopotentials. Phys. Rev. Lett. 48, 1425-1428 (1982). 
517 [40] Louie, S. G., Froyen, S. \& Cohen, M. L. Nonlinear ionic pseudopotentials in spin-densityfunctional calculations. Phys. Rev. B 26, 1738-1742 (1982).

519 [41] Frisch, M. J. et al. Gaussian 16 Revision C.01 (2016). Gaussian Inc. Wallingford CT. 


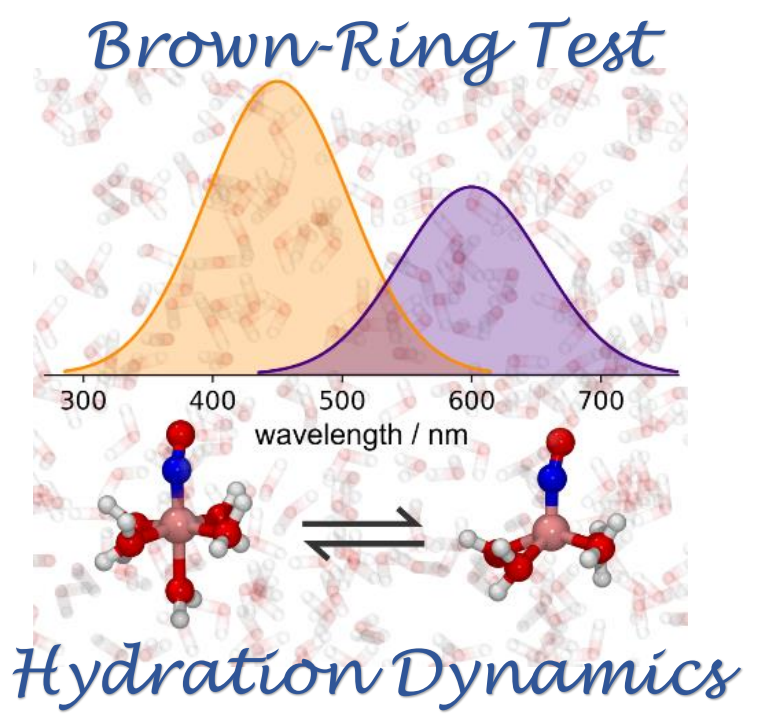

520 